\title{
Amebiasis: aspectos clínicos, terapéuticos y de diagnóstico de la infección
}

\author{
LEONOR CHACÍN-BONILLA
}

\section{An update on amebiasis}

The description of Entamoeba dispar, and the recovery of Entamoeba moshkovskii from humans had a major impact in the epidemiology and clinical management of amebiasis. Infections range from asymptomatic colonization to hemorrhagic colitis and extra-intestinal diseases. Only a minority of amebiasis patients progress to the development of disease. Recent studies suggest that susceptibility to infection, and its outcome is influenced by the host, parasite genotype, and environment. The identification of Entamoeba histolytica is based on the detection of specific antigens by ELISA and DNA in stool and other clinical samples. Several diagnostic tests have been developed, including polymerase chain reaction, the technique of choice, for the detection and differentiation of E. histolytica, E. dispar, and E. moshkovskii. Combination of serologic tests with detection of the parasite DNA by PCR or antigen by ELISA offers the best approach to diagnosis. However, these techniques are impractical for clinical laboratories of developing countries. Clinicians must follow the guidelines of the World Health Organization to avoid unnecessary treatments. This review describes and discusses recent advances in amebiasis with emphasis in the clinical aspects and management of infection.

(Rev Med Chile 2013; 141: 609-615).

Key words: Amebiasis; Diagnosis; Entamoeba histolytica; Epidemiology; Treatment.

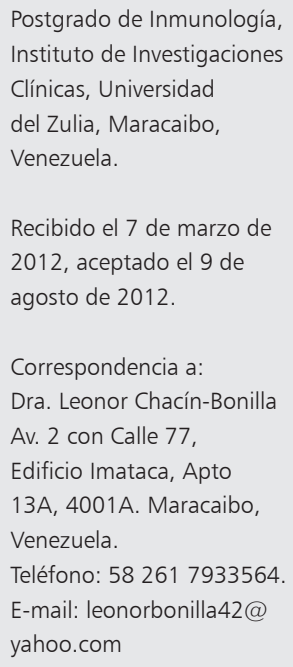
Instituto de Investigaciones Clínicas, Universidad del Zulia, Maracaibo, venezuela.

Recibido el 7 de marzo de 2012, aceptado el 9 de agosto de 2012.

Correspondencia a: Dra. Leonor Chacín-Bonilla Av. 2 con Calle 77, Edificio Imataca, Apto 13A, 4001A. Maracaibo, Venezuela. Teléfono: 582617933564. E-mail: leonorbonilla42@ yahoo.com

L a amebiasis se considera como una causa importante de morbilidad y mortalidad en el mundo y constituye dentro de las enfermedades de origen parasitario, especialmente en el mundo en desarrollo, la tercera causa de muerte después de la malaria y la esquistosomiosis ${ }^{1,2}$. El reconocimiento de Entamoeba dispar y Entamoeba moshkovskii en humanos ha complicado más el diagnóstico de E. histolytica. Estas tres especies son morfológicamente indistinguibles y las dos primeras se consideran comensales. Sin embargo, estudios recientes sugieren que puedan desempeñar un papel patógeno. En Bangladesh e India, $E$. dispar y E. moshkovskii se observaron asociadas con síntomas gastrointestinales ${ }^{3}$ y se ha demostrado que $E$. dispar produce lesiones intestinales y hepáticas en conejillos de Indias ${ }^{4}$. La distribución mundial y la magnitud de la infección de estas tres especies no son conocidas, ya que la mayoría de los diagnósticos, especialmente en el tercer mundo, se siguen basando en la observación microscópica lo que no permite diferenciarlas. Los datos actuales sugieren que E. dispar es 10 veces más común que $E$. histolytica en individuos asintomáticos pero las prevalencias locales de ambas especies varían significativamente ${ }^{1}$. No todas las infecciones con E. histolytica conducen a la enfermedad y sólo una de cada 10 infecciones progresan hacia la enfer$\operatorname{medad}^{3}$. El objetivo de esta revisión es describir y discutir los avances recientes en la amebiasis con énfasis en los aspectos clínicos, de diagnóstico y manejo de la infección.

\section{La epidemiología de la amebiasis}

La amebiasis es común y ampliamente diseminada, pero la prevalencia varía según las áreas geográficas. Alrededor de 50 millones de personas 
al año se infectan con el parásito; no obstante, sólo 5 millones (10\%) desarrollan la enfermedad, resultando en 100.000 muertes anuales ${ }^{2,5}$. La prevalencia mundial de E. histolytica no es bien conocida, por el surgimiento de E. dispar y E. moshkovskii como especies que infectan al hombre. En países en vías de desarrollo se han realizado pocos estudios al respecto. El rango de la prevalencia es de 1\% a 40\% en América Central y del Sur, Asia y África y de $0,2 \%$ a $10,8 \%$ en países industrializados ${ }^{6,7}$. En México ${ }^{8}$, Brasil ${ }^{7}$, Nicaragua ${ }^{9}$ y Ecuador ${ }^{10}$, se han observado porcentajes de infección con $E$. histolytica de $0 \%$ a $13,8 \%$ y de E. dispar de $7,5 \%$ a 2,8\%. En Bangladesh, se demostraron nuevas infecciones por E. histolytica en 39\% de los niños estudiados durante un año, de los cuales $10 \%$ desarrolló diarrea y $3 \%$ disentería $^{11}$.

Gran parte de la morbilidad y mortalidad ocasionadas por la infección ocurre en las regiones en desarrollo ${ }^{12}$. Estudios realizados en México demostraron la presencia de anticuerpos contra E. histolytica en $8,4 \%$ de la población ${ }^{13}$, mientras que en barrios de Fortaleza, Brasil, 25\% de los individuos estudiados y 40\% de los niños de 6 a 14 años de edad eran seropositivos ${ }^{14}$. En el estado Zulia, Venezuela, se observaron porcentajes de seropositividad al parásito de $4,4 \%$ a $46,6 \%{ }^{15-18}$. En un estudio longitudinal, durante 8 años, en Bangladesh, 90\% de los niños estudiados se infectaron con E. histolytica por lo menos una vez y alrededor de 50\% mostraron evidencias serológicas del parásito alrededor de los 5 años de edad ${ }^{19}$. Estudios realizados por Stauffer y col. (2006) en Sudáfrica y Egipto, demostraron una prevalencia de amebiasis de $15 \%$ a $70 \%{ }^{20}$.

En los países desarrollados, los grupos de alto riesgo a la infección son los viajeros, inmigrantes o visitantes de áreas endémicas, residentes en instituciones para discapacitados y hombres homosexuales que practican el sexo oro-anal ${ }^{12}$. Según algunos autores, los individuos con el virus de la inmunodeficiencia humana (VIH) también son un grupo de riesgo ${ }^{12}$. Sin embargo, en los países del Occidente, aunque de $20 \%$ a $30 \%$ de los hombres homosexuales están infectados con E. dispar, la amebiasis invasiva es muy rara en pacientes con el VIH ${ }^{21}$. En dos estudios realizados en México, ninguno de los pacientes VIH positivos infectados con E. histolytica presentaba síntomas atribuibles al parásito ${ }^{22}$. No obstante, la infección y la enfermedad son relativamente frecuentes en estos pacientes en otros países ${ }^{12,23}$. Estos resultados contradictorios pudieran deberse a la existencia de diferentes genotipos del parásito o a diferentes grados de inmunosupresión de los pacientes.

La infección por E. histolytica se transmite a través de agua, alimentos y manos contaminadas con los quistes, donde los manipuladores de alimentos y los vectores mecánicos son posibles fuentes de infección ${ }^{24}$. La relación sexual oro-anal entre hombres homosexuales ha sido reconocida como un modo de transmisión ${ }^{12,25}$. Los perros y primates podrían ser fuentes potenciales de infección humana. Sin embargo, la transmisión a través de los primeros es improbable y a través de los segundos parece ser mínima ${ }^{25,26}$.

\section{Patogenicidad}

E. histolytica se comporta más frecuentemente como un comensal; el 90\% de las infecciones son asintomáticas ${ }^{27}$. Los factores que determinan su patogenicidad no son bien conocidos. Un estudio prospectivo en niños de Bangladesh sugiere que la susceptibilidad a la infección y su desenlace están influenciados por factores dependientes del hospedero, del parásito y del ambiente ${ }^{19}$.

No todos los niños son susceptibles a la infección y algunos son resistentes ${ }^{11}$. La desnutrición aumenta la susceptibilidad ${ }^{28}$. Los niños desnutridos tienen bajos niveles de la hormona leptina que es responsable de la saciedad e influencia el sistema inmune $^{29}$. El aumento de la susceptibilidad a la infección intestinal está asociada con el polimorfismo genético del receptor de la leptina ${ }^{30}$; los niños con el alelo para la arginina (223R) son casi cuatro veces más propensos a sufrir la infección que los homocigotos para el alelo glutamina $(223 \mathrm{Q})^{29}$. Hay evidencias que los alelos de los antígenos de los leucocitos humanos (ALH) clase II influencian la infección ya que el alelo $\mathrm{DQB} 1^{\star} 0601$ protege contra ésta ${ }^{30}$. También el absceso hepático amebiano (AHA) es más frecuente en hombres ${ }^{24}$. Estudios realizados en ratones sugieren que el aumento del interferón- $\gamma$ y las células T citotóxicas en las mujeres pudieran determinar la resistencia al $\mathrm{AHA}^{31}$, habiéndose observado además que, los sueros de hombres y mujeres difieren en la habilidad de lisar a E. histolytica in vitro ${ }^{32}$.

Pareciera que el genotipo del parásito también influencia el desenlace de la infección ya que éste 
varía significativamente entre pacientes con amebiasis intestinal y aquellos con AHA. El genotipo 66 es más frecuente en pacientes con diarrea o disentería que en aquellos asintomáticos o con AHA y al parecer, sólo ciertos genotipos son capaces de causar el $\mathrm{AHA}^{33}$.

Es posible que el microbioma intestinal influencie el curso de la infección. Estudios in vitro sugieren que la virulencia del parásito depende del tipo de bacteria existente en el cultivo ${ }^{29}$.

La potente actividad citotóxica de E. histolytica parece ser el factor fundamental de la patogénesis del parásito, la que ha sido atribuida a la capacidad de la ameba de destruir los tejidos a través de la adherencia a las células blanco, apoptosis y proteólisis de la matriz extracelular del hospede$\mathrm{ro}^{34}$. Sin embargo, otros factores contribuyen en la destrucción celular: 1 . Una lectina del parásito media su unión a la galactosa (Gal) y la N-acetilD-galactosamina (GalNAc) de las células blanco, lo cual es crítico en la adherencia y citotoxicidad del parásito; 2. Los amebaporos que inducen la formación de poros en liposomas sintéticos, pero se desconocen sus funciones específicas; 3 . Las cisteíno proteasas que actúan sobre varios sustratos del hospedero y 4 . Las proteínas de la membrana que son efectores potenciales ${ }^{29}$.

Estudios realizados en modelos animales que reproducen el AHA han demostrado que la respuesta inflamatoria del hospedero es el factor fundamental del daño tisular. Pareciera que la virulencia del parásito está determinada primordialmente por su capacidad de adaptación y sobrevivencia en el ambiente aeróbico del tejido. Este período inicial en la relación hospederoparásito aparenta ser un requisito para continuar produciendo las lesiones, las cuales son producto de la acción conjunta de moléculas provenientes del hospedero y del parásito ${ }^{34}$.

\section{Cuadro clínico de la amebiasis}

La amebiasis intestinal se caracteriza clásicamente por disentería y dolor abdominal. También puede ocurrir diarrea acuosa o con moco abundante. Histológicamente, se pueden observar los trofozoítos en la pared intestinal y las típicas úlceras en forma de botella. El colon ascendente es la región del intestino grueso más afectada. La colitis grave se manifiesta con disentería severa, dolor abdominal y raramente fiebre. La colitis necrotizante extensa es a menudo fatal ${ }^{24}$. Los grupos con mayor riesgo de tener una evolución grave son los niños, ancianos, desnutridos y los pacientes que reciben terapia con corticoides ${ }^{12}$. Las complicaciones incluyen estrechez u obstrucción intestinal, fístula rectovaginal, ameboma, megacolon tóxico, ulceración perianal y perforación intestinal con peritonitis, shock y deceso ${ }^{35}$. Se ha descrito la amebiasis intestinal crónica con diarrea y dolor abdominal intermitentes y períodos de constipación ${ }^{24}$.

La expresión clínica extraintestinal más frecuente es el AHA debido a la diseminación hematógena de las amebas desde el colon al hígado vía la vena porta lo que explica la mayor frecuencia del absceso en el lóbulo derecho del órgano ${ }^{36}$. En la mayoría de estos casos, no se detecta infección intestinal concomitante. Los adultos jóvenes son los más afectados y la afección se puede presentar incluso a los meses o años después de la exposición al parásito ${ }^{24}$. El cuadro clínico se caracteriza por fiebre, escalofríos, sudor, dolor abdominal y hepatomegalia sensible a la palpación. Puede haber tos y estertores en la base del pulmón derecho. La ictericia es inusual. Los síntomas son generalmente agudos, pero pueden ser crónicos acompañados de anorexia y pérdida de peso. Los hallazgos más comunes de laboratorio son leucocitosis sin eosinofilia, anemia, eritrosedimentación elevada y aumento de la fosfatasa alcalina. La bilirrubina está elevada en menos de $50 \%$ de los casos. Las complicaciones incluyen infección bacteriana, ruptura del absceso hacia la cavidad pleural, pericardial y peritoneal, shock séptico y muerte. Pueden ocurrir, raramente, metástasis amebianas hacia otros órganos ${ }^{35}$.

\section{El diagnóstico de laboratorio}

Las técnicas de PCR se han convertido en los métodos de elección por su excelente sensibilidad y especificidad en el diagnóstico de la infección y por la detección y capacidad para diferenciar las tres especies de Entamoeba morfológicamente indistinguibles ${ }^{35}$. La aplicación de estas técnicas es factible principalmente en países industrializados, donde la amebiasis afecta especialmente algunos grupos de alto riesgo ${ }^{12}$. En los países con escasos recursos, estas técnicas han sido poco utilizadas 
por su complejidad, costo y desconocimiento. Es necesario entrenar personal en el manejo de esta metodología y crear conciencia en los médicos de la necesidad de utilizar las técnicas moleculares en el diagnóstico de la infección.

En el tercer mundo, la detección de antígenos de E. histolytica en heces por ELISA, mediante el kit de E. histolytica II generación (Tech Lab), es una buena opción para el diagnóstico en laboratorios clínicos donde no sea viable la utilización de los métodos moleculares ${ }^{3}$. La combinación de esta prueba con técnicas serológicas ofrece la mejor metodología para el diagnóstico de los casos clínicos. La limitación de estas técnicas es la inhabilidad para diferenciar las infecciones actuales de las pasadas, lo que dificulta el diagnóstico clínico ${ }^{3,27}$. De estas pruebas, ELISA es la más usada y es suficiente para fines clínicos, especialmente para el diagnóstico de AHA. Se ha demostrado que un kit comercial de microtitutación por ELISA (LMD Laboratories Inc. Carisbad, CA, USA) tiene una sensibilidad de $97,9 \%$ y una especificidad de $94,8 \%$ para la detección de anticuerpos a $E$. histolytica en pacientes con $\mathrm{AHA}^{3}$.

En el mundo en desarrollo, el microscopio continúa siendo la piedra angular de los microscopistas y consecuentemente, de los diagnósticos erróneos de E. histolytica / E. dispar (Eh/Ed), que continúan siendo frecuentes 9 . Sin embargo, existen procedimientos que permiten la identificación microscópica de Eh/Ed. Uno de los requisitos primarios para el diagnóstico correcto es el examen de una serie de especímenes fecales muy frescos. El material que se vaya a examinar debe ser estudiado mediante los siguientes métodos: 1 . Frotis frescos con solución salina y de yodo; 2 . Un método de concentración confiable y 3 . Frotis teñidos con una tinción permanente. El riesgo de cometer errores de diagnóstico es alto cuando se basa sólo en frotis frescos (directos o concentrados). La diversidad de especies de amebas, células y otros elementos que se pueden observar en las heces, ocasiona un cuadro complejo y muchas células pueden ser confundidas con Eh/Ed en preparaciones al fresco. Además, amebas pequeñas como Entamoeba hartmanni y Endolimax nana podrían no ser detectadas, o sus rasgos morfológicos ser indistinguibles. Entamoeba polecki, morfológicamente similar a $E h / E d$, podría pasar desapercibida. El diagnóstico diferencial microscópico de Eh/Ed depende de rasgos citológicos finos por lo que no es adecuado su diagnóstico sin el concurso de una tinción permanente ${ }^{24}$. Consideramos que la tinción con hematoxilina férrica es un método crucial, confiable y el más crítico en el diagnóstico microscópico de $E h / E d^{37}$.

\section{Tratamiento}

Se recomienda tratar todos los casos de infección con E. histolytica. Cuando no es posible diferenciar esta ameba de otras morfológicamente iguales se recomienda no tratar a los casos asintomáticos ${ }^{1}$. La selección de la droga y su vía de administración, se basa en la localización de los trofozoítos en la luz o pared intestinal o en tejidos extraintestinales. Otros factores a considerar son: la edad, severidad clínica, presencia de otros parásitos intestinales o condiciones mórbidas, disponibilidad de los fármacos, eficacia clínica y parasitológica de las drogas, efectos colaterales y la existencia de embarazo ${ }^{38}$.

Tratamiento de la amebiasis intestinal asintomática. Se trata con amebicidas luminales. La paramomicina se administra a dosis de $30 \mathrm{mg} / \mathrm{kg}$, divididos en tres dosis, durante 10 días $^{39}$. El furoato de diloxanida se prescribe a dosis de $500 \mathrm{mg}$ t.i.d. durante 10 días y la dicloroacetamida (teclozan) se administra, durante 5 días, a dosis de $500 \mathrm{mg}$ b.i.d., para un total de $1.500 \mathrm{mg}$ en un día, para adultos y niños $>8$ años; de $50 \mathrm{mg}$ t.i.d. para niños de 3 a 8 años y de 25 mg t.i.d. para niños de 1 a 3 años ${ }^{38}$.

Tratamiento de la amebiasis intestinal invasiva. Los 5-nitroimidazoles, particularmente el metronidazol (MTZ), son las drogas de elección para el tratamiento de la infección tisular; ya que alrededor de $90 \%$ de los pacientes con disentería leve o moderada responden a estos fármacos ${ }^{39}$. El MTZ es la droga más usada a dosis de $750 \mathrm{mg}$ t.i.d. durante 5 a 10 días, dependiendo de la severidad de la sintomatología ${ }^{40}$. Este tratamiento debe ser seguido con la administración de un agente que actúe en la luz intestinal. En los casos de colitis fulminante, se puede administrar la droga por vía parenteral y se recomienda dar antibióticos de amplio espectro para atacar las bacterias intestinales que pueden invadir el peritoneo. Estos pacientes pueden necesitar cirugía por abdomen agudo, sangrado intestinal o magacolon tóxico ${ }^{39}$.

Tratamiento de la amebiasis extraintestinal. En el AHA, el MTZ es la droga de elección a dosis 
de $750 \mathrm{mg}$ t.i.d. durante 5 a 10 días seguida de la administración de un amebicida luminal ${ }^{40}$. El drenaje percutáneo o quirúrgico del absceso no se recomienda por el peligro potencial de infección bacteriana o ruptura. Sin embargo, la aspiración terapéutica a veces es necesaria como terapia adyuvante como en los casos de falta de respuesta a la droga antiparasitaria a los 4 ó 5 días, ruptura inminente e infección bacteriana. El tratamiento percutáneo es el procedimiento de elección para la reducción del tamaño del absceso ${ }^{39}$. La ultrasonografía puede ser usada para monitorear la regresión del absceso después del tratamiento, que ocurre de 3 a 12 meses $^{36}$. En relación a la terapia de la amebiasis en otros órganos, no existen pautas definidas ya que la mayoría de estos casos son muy raros. En el caso de la amebiasis pulmonar, la farmacoterapia es igual a la del AHA $^{41}$.

Los 5-nitroimidazoles continúan siendo importantes en la terapia de la amebiasis y el MTZ sigue siendo la droga de elección. Sin embargo, este fármaco tiene efectos tóxicos ${ }^{39,42}$ y se han reportado fracasos en el tratamiento de algunas bacterias y protozoos $^{43,44}$, pero no se han observado aislados de E. histolytica resistentes al fármaco ${ }^{45}$. Por estas razones se hace necesaria la búsqueda de nuevos fármacos amebicidas. La nitazoxanida (NTZ) es una nueva droga que tiene una actividad de amplio espectro incluyendo algunas bacterias y diversos protozoos y helmintos ${ }^{46}$. Esta droga es prometedora para el tratamiento de la infección y ya se ha demostrado su actividad contra E. histolytica in vitro y en humanos con la infección y diarrea ${ }^{39}$. Este fármaco podría ser clave como amebicida por su efectividad contra los parásitos tanto en los tejidos como en la luz intestinal siendo importante además considerar su amplio espectro.

\section{Prevención}

El mejoramiento de las condiciones de vida, incluyendo viviendas adecuadas, suministro de agua potable, eliminación apropiada de las heces, higiene personal y la educación sanitaria de la población, contribuyen a evitar la transmisión y adquisición de la infección. Los manipuladores de alimentos deben ser examinados, controlados regularmente y tratados en caso de estar infecta$\operatorname{dos}^{24,38}$. En hombres homosexuales, se debe evitar el contacto sexual oro-anal.
La persistencia de la morbilidad asociada a la amebiasis sugiere que las estrategias para minimizar su transmisión no han sido efectivas. Un programa de control adecuado a corto plazo, es difícil de implementar, porque implica mejorar las condiciones de vida de un gran segmento de la población mundial. Por ello, el diseño de una vacuna para la prevención de la amebiasis es muy deseable. Se han logrado vacunas que proveen altos porcentajes de protección contra la enfermedad en animales de experimentación ${ }^{47,48}$. Sin embargo, la mayoría de los adyuvantes usados no son aceptables para su uso en humanos y se desconoce la efectividad de las respuestas inmunes para prevenir la enfermedad en el hombre ${ }^{49}$.

\section{Conclusiones}

Es necesario utilizar técnicas que permitan diferenciar E. histolytica, E. dispar y E. moshkovskii para poder realizar el diagnóstico de amebiasis y redefinir la epidemiología global de la infección. Las técnicas de PCR son las de elección en el diagnóstico de laboratorio. En áreas donde estas técnicas no sean accesibles, la detección de antígenos de E. histolytica por ELISA es una buena opción y su combinación con técnicas serológicas ofrecen buenas opciones metodológicas para el diagnóstico clínico.

Desde el punto de vista terapéutico, todos los casos de amebiasis deben ser tratados. Si el diagnóstico es microscópico, se tratan sólo los casos sintomáticos. Es indispensable la búsqueda de otras drogas amibicidas y es deseable el diseño de una vacuna para prevenir la infección.

\section{Referencias}

1. WHO/PAHO/UNESCO. A consultation with experts on amoebiasis. Epidemiol Bull 1997; 18 (1): 13-4.

2. Walsh JA. Problems in recognition and diagnosis of amebiasis: estimation of the global magnitude of morbidity and mortality. Rev Infect Dis 1986; 8: 228-38.

3. Chacín-Bonilla L. Amibiasis: Implicaciones del reconocimiento de Entamoeba dispar e identificación de Entamoeba moshkovskii en humanos. Invest Clin 2010; 51 (2): 239-56.

4. Costa CA, Brito KN, Gómes MA, Caliari MV. Histopathological and immunohistochemical study of the 
hepatic lesions experimentally induced by Entamoeba dispar. Eur J Histochem 2010; 54 (e39): 170-4.

5. Jackson TF. Entamoeba histolytica and Entamoeba dispar are distinct species; clinical, epidemiological and serological evidence. Int J Parasitol 1998; 28: 181-6.

6. Rivera WL, Tachibana H, Kanbara H. Field study on the distribution of Entamoeba histolytica Entamoeba dispar in the Northern Philippines as detected by the polimerase chain reaction. Am J Trop Med Hyg 1998; 59 (6): 916-21.

7. Braga LL, Mendonca Y, Paiva CA, Sales A, Cavalcante AL, Mann BJ. Seropositivity for and intestinal colonization with Entamoeba histolytica and Entamoeba dispar in individuals in Northeastern Brazil. J Clin Microbiol 1998; 36 (10): 3044-5.

8. Ramos F, Morán P, González E, García G, Ramiro M, Gómez A, et al. High prevalence rate of Entamoeba histolytica asymptomatic infection in a rural Mexican community. Am J Trop Med Hyg 2005; 73 (1): 87-91.

9. Leiva B, Lebbad M, Winiecka-Krusnell J, Altamirano I, Téllez A, Linder E. Overdiagnosis of Entamoeba histolytica and Entamoeba dispar in Nicaragua. A microscopic, triage parasite panel and PCR study. Arch Med Res 2006; 37 (4): 529-34.

10. Levecke B, Dreesen L, Barrionuevo-Samaniego M, Benítez Ortíz W, Praet N, Brandt J et al. Molecular differentiation of Entamoeba spp. in a rural community of Loja province, South Ecuador. Trans R Soc Trop Med Hyg 2011; 105: 737-9.

11. Haque R, Mondal D, Duggal P, Kabir M, Roy S, Far BM et al. Entamoeba histolytica infection in children and protection from subsequent amebiasis. Infect Immun 2006; 74 (2): 904-9.

12. Tanyuksel M, Petri WA Jr. Laboratory diagnosis of amebiasis. Clin Microbiol Rev 2003; 16 (4): 713-9.

13. Caballero-Salcedo A, Viveros-Rogel M, Salvatierra B, Tapia-Conyer R, Sepúlveda-Amor J, Gutiérrez G. Seroepidemiology of amebiasis in México. Am J Trop Med Hyg 1994; 50 (4): 412-9.

14. Braga LL, Lima AM, Sears CL, Newman RD, Wuhib T, Paiva CA, et al. Seroepidemiology of Entamoeba histolytica in a slum in northeastern Brazil. Am J Trop Med Hyg 1996; 55 (6): 693-7.

15. Chacín-Bonilla L, Bonpart D. A seroepidemiological study of amebiasis in adults in Venezuela. Am J Trop Med Hyg 1981; 30 (6): 1201-5.

16. Chacín-Bonilla L, Chacín-Martínez E, Espinoza E, Cárdenas B. A seroepidemiological study of amebiasis in children of low socioeconomic level in Maracaibo, Venezuela. Am J Trop Med Hyg 1982; 31 (6): 1103-6.

17. Chacín-Bonilla L, Mathews HM, Gealy GR, Dikdan Y,
Rodríguez-Zambrano N. Serologic and parasitological studies of amebiasis in two suburban communities of Maracaibo, Venezuela. Invest Clin 1984; 25 (2): 69-80.

18. Chacín-Bonilla L, Mathews HM, Dikdan Y, Guanipa N. Estudio seroepidemiológico de la amibiasis en una comunidad del estado Zulia, Venezuela. Rev Inst Med Trop Sao Paulo 1990; 32 (6): 467-3.

19. Petri WA Jr, Mondal D, Peterson KM, Duggal P, Haque R. Association of malnutrition with amebiasis. Nutr Rev 2009; 67 (Suppl. 2): S207-15.

20. Stauffer W, Abd-Alla M, Ravdin JI. Prevalence and incidence of Entamoeba histolytica infection in South Africa and Egypt. Arch Med Res 2006; 37: 266-9.

21. Hung CC, Deng HY, Hsiao WH, Hsieh SM, Hsiao CF, Chen MY, et al. Invasive amoebiasis as an emerging parasitic disease in patients with human immunodeficiency virus type-1 infection in Taiwan. Arch Inter Med 2005; 165: 409-5.

22. Morán P, Gómez A, Valadez A, García G, Ramos F, González E, et al. Periodicity and patterns of Entamoeba histolytica and Entamoeba dispar infection in HIV+l AIDS patients in Mexico. Ann Trop Med Parasitol 2009; 103 (4): 307-15.

23. Haghihgi A, Kobayashi S, Takeuchi T, Thammapalerd N, Nozaki T. Geographic diversity among genotypes of Entamoeba histolytica field isolates. J Clin Microbiol 2003; 4: 3748-56.

24. Beaver PC, Jung RC, Cupp EW. Parasitología Clínica. Editores Salvat, Barcelona, España. 1986; 882 pp.

25. Schuster FL, Visvesvara GS. Amebae and ciliated protozoa as causal agents of waterborne zoonotic disease. Vet Parasitol 2004; 126: 91-120.

26. Thompson RCA. The zoonotic significance and molecular epidemiology of Giardia and giardiasis. Vet Parasitol 2004; 126: 15-35.

27. Gathiram V, Jackson TF. A longitudinal study of asymptomatic carriers of pathogenic zimodemes of Entamoeba histolytica. S Afr Med J 1987; 72 (10): 669-72.

28. Mondal D, Petri WA Jr, Sack RB, Kirkpatrick BD, Haque R. Entamoeba histolytica-associated diarrheal illness is negatively associated with the growth of preschool children: evidence from a prospective study. Trans R Soc Trop Med Hyg 2006; 100: 1032-8.

29. Ralston KS, Petri WA Jr. Tissue destruction and invasion by Entamoeba histolytica. Trends Parasitol 2011; 27 (6): 253-62.

30. Duggal P, Guo X, Haque R, Peterson KM, Ricklefs S, Mondal D. A mutation in the leptin receptor is associated with Entamoeba histolytica infection in children. J Clin Invest 2011; 121: 1191-8.

31. Lotter H, Jacobs T, Gaworski I, Tannich E. Sexual dimor- 
phism in the control of amebic liver abscess in a mouse model of disease. Infect Immun 2006; 74: 118-24.

32. Snow M, Chen M, Guo J, Atkinson J, Stanley SL Jr. Differences in complement-mediated killing of Entamoeba histolytica between men and women-an explanation for the increased susceptibility of men to invasive amebiasis? Am J Trop Med Hyg 2008; 78 (6): 922-3.

33. Ali IK, Solaymani-Mohammadi S, Akhter J, Roy S, Gorrini C, Calderaro A, et al. Tissue invasion by Entamoeba histolytica: Evidence of genetic selection and/or DNA reorganization events in organ tropism. PLoS Negl Trop Dis 2008; 2 (6): e219.

34. Olivos-García A, Saavedra E, Ramos-Martínez E, Nequiz M, Pérez-Tamayo R. Molecular nature of virulence in Entamoeba histolytica. Infect Genet Evol 2009; 9: 1033-7.

35. Fotedar R, Stark D, Beebe N, Marriott D, Ellis J, Harkness J. Laboratory diagnostic techniques for Entamoeba species. Clin Microbiol Rev 2007; 20 (3): 511-32.

36. Salles JM, Moraes LA, Salles MC. Hepatic amebiasis. Braz J Infect Dis 2003; 7 (2): 96-110.

37. Chacín-Bonilla L. Diagnóstico microscópico de amibiasis: Método obsoleto pero necesario en el mundo en desarrollo. Invest Clin 2011; 52 (4): 291-4.

38. Botero DA, Restrepo M. Parasitosis humana. Editorial Presencia Ltda, Bogotá, Colombia. 1992; 418 pp.

39. Petri WA Jr. Therapy of intestinal protozoa. Trends Parasitol 2003; 19 (11): 523-6.

40. Bobbi S, Pritt MD, Clark G. Amebiasis. Mayo Clin Proc 2008; 83 (10): 1154-60.

41. Knight R. The chemotherapy of amoebiasis. J Antimicrob Chemother 1980; 6: 577-593.

42. Hayat F, Salahuddin A, Zargan J, Azam A. Synthesis, characterization, antiamoebic activity and cyto toxicity of novel 2-(quinolin-8-yloxy) acetohydrazones and their cyclized products (1,2,3- thiadiazole and 1,2,3-selenadiazole derivatives). Eur J Med Chem 2010; 45: 6127-34.

43. Escobedo AA, Cimerman S. Giardiasis: a pharmacotherapy review. Expert Opin Pharmacother 2007; 8 (12): 1885-902.

44. Fujishima T, Nishise S, Ichihara M, Kobayashi S, Takeuchi T. Difficulties in the treatment of intestinal amoebiasis in mentally disabled individuals at a rehabilitation institution for the intellectually impaired in Japan. Chemotherapy 2010; 56: 348-52.

45. Ali V, Nozaki T. Current therapeutics, their problems, and sulfur-containing-amino-acid metabolism as a novel target against infections by "Amitochondriate" protozoan parasites. Clin Microbiol Rev 2007; 20 (1): 164-87.

46. Gilles H, Hoffman PS. Treatment of intestinal parasitic infections: a review of nitazoxanide. Trends Parasitol 2002; 18 (3): 95-7.

47. Carrero JC, Contreras-Rojas A, Sánchez-Hernández B, Petrosyan P, Bobes RJ, Ortíz-Ortíz L, et al. Protection against murine intestinal amoebiasis induced by oral immunization with the $29 \mathrm{kDa}$ antigen of Entamoeba histolytica and cholera toxin. Exp Parasitol 2010; 126 (3): 359-65.

48. Guo X, Barroso L, Lyerly DM, Petri WA Jr, Houpt ER. CD4+ and CD8+ T cell- and IL-17-mediated protection against Entamoeba histolytica induced by a recombinant vaccine. Vaccine 2011; 29: 772-7.

49. Stanley SL Jr. Vaccines for amoebiasis: Barriers and opportunities. Parasitology 2006; 133 (Suppl.): S81-6. 